\title{
Non-linear rheological behavior of gluten-free flour doughs and correlations of LAOS parameters with gluten-free bread properties
}

\author{
Gamze Yazar $^{\text {a, b }}$, Ozlem Duvarci ${ }^{\text {a, c }}$, Sebnem Tavman ${ }^{\text {b }}$, Jozef L. Kokini a, * \\ a Purdue University Food Science Department, 745 Agriculture Mall Dr, West Lafayette, IN 47907, USA \\ ${ }^{\mathrm{b}}$ Ege University Food Engineering Department, Ege University Campus, Bornova, Izmir, Turkey \\ ${ }^{\mathrm{c}}$ Izmir Institute of Technology, Department of Chemical Engineering, Urla, Izmir, Turkey
}

\section{A R T I C L E I N F O}

\section{Article history:}

Received 2 July 2016

Received in revised form

28 December 2016

Accepted 17 January 2017

Available online 27 January 2017

\section{Keywords:}

LAOS

Lissajous-Bowditch curves

Gluten-free dough

Water absorption

Loaf volume

\begin{abstract}
A B S T R A C T
Predicting loaf volume development of gluten free baked products to have similar properties to wheat products remains a challenge and there is no good marker for loaf volume. Large Amplitude Oscillatory Shear (LAOS) flow experiments and baking tests were conducted on rice, buckwheat, quinoa, and soy flour doughs to understand if there is any correlation between the non-linear rheological properties and loaf volume. The challenging water absorption capacities were determined by matching the $\eta^{*}$ vs. frequency data of the gluten free flours with that of the soft wheat flour dough with moisture content at 500 BU. $110 \%, 90 \%, 85 \%$, and $160 \%$ water levels were found as optimal for rice, buckwheat, quinoa, and soy flour, respectively. The comparison of elastic Lissajous-Bowditch curves showed that the stronger nonlinearities were seen at low frequencies and the wider the loop, the weaker the structure and the more structural breakdown with an order of soft wheat, soy, buckwheat, quinoa and rice flour doughs. Secondary loops have been observed in viscous Lissajous-Bowditch curves which are related to the strong non-linearities in elastic stress. The distributions of elastic and viscous LAOS parameters showed that soy dough has the closest rheological performance to wheat dough among other dough samples, which has the highest protein content. $G_{L}^{\prime}$ and $G_{M}^{\prime}$ values at $10 \mathrm{rad} / \mathrm{s}$ and $200 \%$ strain showed the best correlation among all LAOS parameters with the loaf volume. The strain stiffening/softening property $e_{3} /$ $\mathrm{e}_{1}$ complemented the mechanistic explanations which were offered using $G_{L}^{\prime}$ and $G_{M}^{\prime}$ values.
\end{abstract}

(c) 2017 Elsevier Ltd. All rights reserved.

\section{Introduction}

Gluten is the main network-forming protein in flour, and is responsible for imparting the desired viscoelasticity to dough (Huang and Kokini, 1993). It helps dough retain gas to obtain the desired volume greatly influencing the texture of bread and other cereal products and contributes to the appearance and crumb structure of many bakery product (Bagley et al., 1998). Rheological properties of dough were shown to be very important in bread baking (Huang and Kokini, 1993; Janssen et al., 1996). Gluten-free flour alternatives for bakery items such as bread are especially challenging, since without gluten it is very difficult to obtain the characteristic spongy honeycomb structure of bread. When gluten is absent the bread becomes dense, crumbly, cannot retain its cellular structure and has a poor mouthfeel (Crockett et al., 2011). In

\footnotetext{
* Corresponding author.

E-mail address: jkokini@purdue.edu (J.L. Kokini).
}

order to minimize these problems and improve the spongy structure hydrocolloids/gums (Lazaridou et al., 2007), emulsifiers (Nunes et al., 2009), starches/flours without gluten (Demirkesen et al., 2010), maltodextrin (Witczak et al., 2010), dietary fiber (Gularte et al., 2012) dairy ingredients (Gallagher et al., 2003) and specialized proteins such as soy, whey proteins have been added into the formulations. Water absorption capacity (WAC) in the presence of the other wheat dough constituents has the major effect on dough rheology and on baked product performance and quality (Faubion and Hoseney, 1990). For gluten-free bakery products where gluten hydration properties are not known and a cohesive dough is not formed, this creates a major challenge right at the formulation stage.

The rheology of dough has been studied extensively using small amplitude oscillatory shear rheology (Dus and Kokini, 1990; Amemiya and Menjivar, 1992; Phan-Thien et al., 1997). A lot has been learned from these experiments but, because they only focus on the amplitude of stress and strain $\left(\mathrm{G}^{\prime}\right) /$ strain rate $\left(\mathrm{G}^{\prime \prime}\right)$ as well as the phase angle (tan delta), between the two oscillations much 
intracycle information is ignored. Furthermore, the measured parameters are only valid in the linear region where both stress and strain are perfect oscillations and can be simulated with sinusoidal functions. In the non-linear region, the stress curve is a distorted oscillation and can no longer be simulated with a sinusoidal function. Therefore, Fourier transform rheology along with the use of Chebyshev constants (Ewoldt et al., 2008; Yazar et al., 2016; Duvarci et al., 2016) has offered a new window into a lot of previously untapped information.

The objective of this study is to measure and analyze the nonlinear intracycle rheological properties of the gluten-free bread dough samples prepared using the optimum water absorption levels. The optimum water absorption levels are obtained by matching their $\eta^{*}$ values with the soft wheat flour dough $\eta^{*}$ at the WAC that gives $500 \mathrm{BU}$. Then the intracycle rheology of the gluten free doughs were determined and compared in the nonlinear region using LAOS tests. The new parameters $e_{3} / e_{1}, v_{3} / v_{1}$ and $G_{L}$ obtained through the Lissajous curves with LAOS were compared with the LAOS properties of soft wheat flour dough. We also tested the correlations between the LAOS parameter $\mathrm{G}_{\mathrm{L}}$ with loaf volume of the gluten-free breads as a rheological marker.

\section{Materials and methods}

\subsection{Materials}

Soft wheat flour (12.17\% moisture, $23.9 \%$ wet gluten, $60.2 \%$ water absorption) was obtained from Siemer Milling Company (Hopkinsville, KY). Rice flour (6.48\% moisture), quinoa flour $(10.21 \%$ moisture), and buckwheat flour (11.3\% moisture) were obtained from General Mills (Minneapolis, MN) while soy flour $(8.85 \%$ moisture) was obtained from the National Soybean Research Center at UIUC (Urbana, IL). Salt and the instant dry yeast were obtained from a local market in West Lafayette, IN.

\subsection{Methods}

\subsubsection{Water absorption level}

Farinograph measurements following AACC method 54-21 were not possible because the gluten-free flour dough mass did not adhere to the mixing paddles in the Farinograph. A new method to create equivalence in the rheology was used. In this method, the $\eta^{*}$ vs. frequency of gluten-free flours at different levels of added water were compared with the $\eta^{*}$ vs. frequency of soft wheat flour dough at the WAC which gave a peak at $500 \mathrm{BU}$. Rheological properties were measured using a DHR-3 Rheometer (TA Instruments, USA). Strain sweeps in the strain range of $0.01-100 \%$ at $25^{\circ} \mathrm{C}$ using the frequency of $10 \mathrm{rad} / \mathrm{s}$ were conducted in order to obtain the linear region. Frequency sweeps in the linear region in the range of 0.1-100 rad/s were also conducted. The measurements were carried out once the sample equilibrated to a normal force value of $1 \mathrm{~N}$. The edge of the samples were coated with a thick layer of vacuum grease to prevent moisture loss and time sweeps at a given strain and frequency demonstrated that the sample stayed stable for at least $12 \mathrm{~h}$ without losing moisture.

Four different levels of water were used to determine the optimum water absorption (WA) levels for all the gluten free flour samples. For all flours flour:weight basis values were used. For rice flour $90 \%, 105 \%, 110 \%$, 115\% water; for buckwheat flour $85 \%, 90 \%$, $95 \%, 110 \%$ water; for soy flour $160 \%, 165 \%, 175 \%, 190 \%$ water; for quinoa flour $80 \%, 82 \%, 85 \%, 100 \%$ water were used. Comparison of $\eta^{*}$ values were considered for equivalent WA.

\subsubsection{Dough preparation}

Soft wheat flour dough was prepared using a Farinograph
(Brabender, Germany) according to the AACC method No 54-21 (AACC, 2000). Gluten-free dough samples were prepared by hand mixing, since they could not be prepared using a Farinograph.

\subsubsection{LAOS measurements}

LAOS rheological properties were measured with a DHR-3 Rheometer (TA Instruments, USA) using the LAOS (Large Amplitude Oscillatory Shear Test) mode. The measurements were carried out in triplicate using a $40 \mathrm{~mm}$ sand-blasted plate to minimize slip at a gap of $2 \mathrm{~mm}$ at $25^{\circ} \mathrm{C}$ using 10,1, and $0.1 \mathrm{rad} / \mathrm{s}$ frequency levels and between the strain values of 0.01 and 200\%. Showing superimposition at different gap sizes proved lack of slip. Samples were allowed to relax until the axial normal force was $1 \mathrm{~N}$. The predominantly elastic non-linear properties $S$ (stiffening ratio), $\mathrm{e}_{3} / \mathrm{e}_{1}$ (the ratio of the 3rd order elastic Chebyshev coefficient to the $1 \mathrm{st}$ order elastic Chebyshev coefficient), $\mathrm{G}_{\mathrm{L}}$ (large strain modulus) were obtained using the TRIOS software. Lissajous curves which are plots of intracycle normalized stress vs. strain loops were plotted using the software OriginPro 8.6 at strain points of $0.01 \%, 0.06 \%, 1.6 \%, 11 \%$, $28 \%, 44 \%, 70 \%, 105 \%$, and $200 \%$.

\subsubsection{Baking tests}

The baking tests were conducted using a basic formulation which consisted of $100 \mathrm{~g}$ flour as the basis with $1 \%$ salt and 1.5\% yeast. All the dough samples were prepared using $200 \mathrm{~g}$ flour and mixed with a Kitchen-Aid mixer using the flat beater mixing attachment by mixing for $1 \mathrm{~min}(10 \mathrm{~s}$ stirring, $20 \mathrm{~s} 4$ th level, $20 \mathrm{~s}$ 2nd level). The dough hook attachment was used for the wheat flour dough and the mixing took $4 \mathrm{~min}$ ( $1 \mathrm{~min}$ at the 2 nd level speed, followed by $2 \mathrm{~min}$ at the 4 th level speed, followed by $1 \mathrm{~min}$ at the 2 nd level speed again). An Electrolux oven was used for the fermentation and baking steps. The 1 st fermentation was carried out at $38 \mathrm{C}$ for $45 \mathrm{~min}$ and then the dough samples were taken to the molds (150 $\mathrm{g}$ dough for each loaf) and then the 2nd fermentation was carried out again at $38^{\circ} \mathrm{C}$ for $30 \mathrm{~min}$. Baking was carried out at $177^{\circ} \mathrm{C}$ for $35 \mathrm{~min}$ and all the tests were carried out in duplicates.

\subsubsection{Bread volume}

Bread volume was determined using the AACC method no 10-05.01.

\section{Results and discussion}

\subsection{Water absorption capacities for gluten-free flours}

The complex viscosities $\left(\eta^{*}\right)$ for $110 \%$ water content rice flour, $90 \%$ water content buckwheat flour, $160 \%$ water content soy flour, $85 \%$ water content quinoa flour all superimposed (Fig. 1). This shows the equivalency of these water contents in terms of equalizing the dough rheology for gluten-free flours with soft wheat flour as the basis for comparison.

\subsection{Analysis of Lissajous curves}

Both frequency and magnitude of deformation (strain) dictates the rheological response of a viscoelastic material in the non-linear region and LAOS analysis has the ability to fully explore and understand frequency and magnitude dependence of the viscoelastic behavior in oscillatory flow by presenting Lissajous-Bowditch curves at different strain and frequency. This protocol enables us to capture intracycle structural changes and describe these changes as linear response or strain stiffening/softening and shear thinning/ thickening behaviors in the non-linear region. Consequently, Lissajous curves characterize the intracycle rheology of a material and 


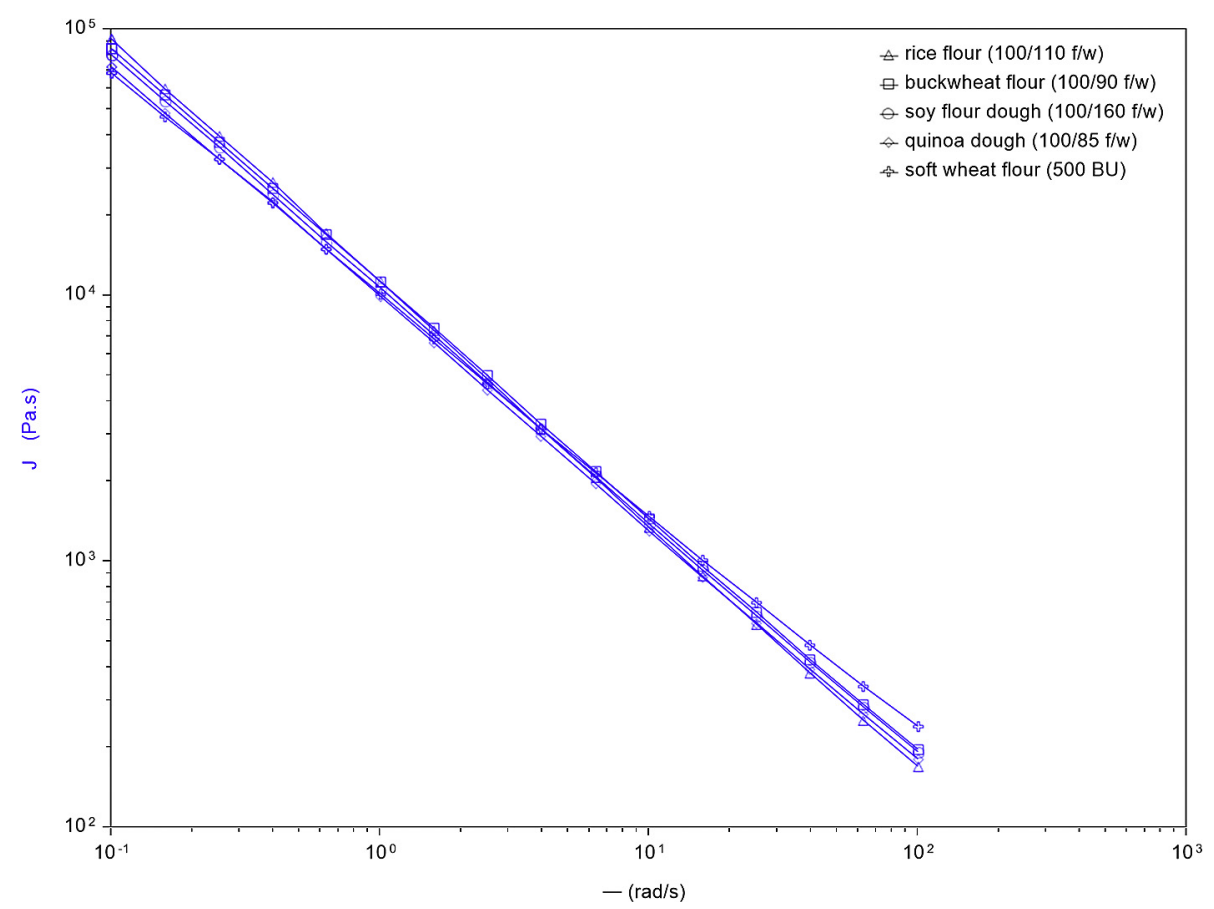

Fig. 1. Comparison of $\eta^{*}$ for gluten-free flours at the optimal water absorption level.

are essential in order to understand the progressive change occurring in the non-linear region and interpret that in terms of possible structural explanations. The normalized plots $\left(\sigma / \sigma_{o}\right.$ versus $\gamma / \gamma_{o}$ or $\left.\dot{\gamma} / \dot{\gamma}_{o}\right)$ give a better understanding of change in stress response with the strain magnitude by adjusting values to a common scale which enables sound comparison of changes in nonlinear intracycle rheological behavior. There is a great deal of new intracycle rheological property information in the Lissajous curves.

\subsubsection{Elastic component}

Circular trajectories in elastic Lissajous curves indicate that the material is predominantly viscous while narrow elliptical trajectories indicate predominantly elastic behavior and elliptical trajectories with finite major to minor axis indicate viscoelastic behavior (Ewoldt et al., 2007). The stress response in the linear region is elliptically shaped and the curve becomes progressively wider and more and more distorted as strain is increased and the material becomes progressively more non-linear as it is seen in the outer loops in Fig. 2. Lissajous curves for the gluten-free dough samples were plotted at 9 selected strain points of $0.01 \%, 0.06 \%$, $1.6 \%, 11 \%, 28 \%, 44 \%, 70 \%, 105 \%$, and $200 \%$. The inner loops at low strain represent the intracycle behavior in the linear region, while the outer loops at higher strains represent the intracycle behavior in the non-linear region. Lissajous curves showed that nonlinearity starts after about $1.6 \%$ strain for all gluten-free dough samples. The elastic Lissajous curves in Fig. 2 showed increasing viscous character as the frequency decreased from $10 \mathrm{rad} / \mathrm{s}$ to $0.1 \mathrm{rad} / \mathrm{s}$ with increasing strain in the non-linear region. The area covered by the loops is related to the energy spent during oscillatory flow. The lower the frequency, the longer time scale for oscillatory flow; hence, the sample has more time to align its structure in the direction of flow. The wider and fatter the loops in the elastic perspective, the higher the energy spent during flow and consequently more viscous behavior suggesting irreversible structure breakdown. When the network is weaker, this transition happens sooner and therefore the Lissajous curves become wider and fatter sooner (at lower strains). This also shows the importance of the energy delivered into the structure of the particular material. The larger the amount of energy, the greater the network and structure breakdown. The most elastic character was observed first for the soft wheat flour dough followed by the soy dough followed by quinoa dough and buckwheat. Rice dough showed the least elastic character as evidenced by the widest elliptical trajectories. Consequently, the rate at which loops became fatter and wider was the highest for rice dough and it followed the reverse order discussed above (see Fig. 3).

Changing time scale of oscillatory deformation (frequency) has very significant effects on the stress response of all gluten free dough samples. The major change for rice flour dough occurs when the frequency decreases from $10 \mathrm{rad} / \mathrm{s}$ to $1 \mathrm{rad} / \mathrm{s}$. Rice dough is considerably less elastic among gluten-free dough samples, because it has the lowest protein content and any network formation is due to hydrogen bonding between starch molecules and consequently the Lissajous curves get wider faster while soy dough (Fig. 2c) demonstrates the most elastic behavior among all glutenfree samples because distorted wide trajectories were not observed. (Fig. 2a). Soy flour dough has the largest protein content and its alcohol soluble 7S fraction resembles gliadin somewhat and its ethanol insoluble 11S fraction $s$ held together with a large number of intermolecular disulfide bonds reminiscent of glutenin behavior. The Lissajous curves for all the other gluten-free dough samples show elliptical trajectories at $10 \mathrm{rad} / \mathrm{s}$ and the trajectories become wider as the frequency decreases to $1 \mathrm{rad} / \mathrm{s}$. The trajectories become distorted when the frequency drops to $0.1 \mathrm{rad} / \mathrm{s}$ evidence of more viscous behavior following the order of rice (Fig. 2a), quinoa (Fig. 2d), buckwheat (Fig. 2b), soy (Fig. 2c) and wheat flour (Fig. 2d) dough samples. This order is similar to the order of protein content of each gluten-free dough samples.

Soy dough shows the least change in the elastic component with the change in frequency (Fig. 2c). We attribute the ability of soy flour dough to impart considerable elasticity to the chemistry of the protein. Soy protein consists of the major fractions the $2 \mathrm{~S}, 7 \mathrm{~S}$ and $11 \mathrm{~S}$ fractions. These interestingly have similar properties to the protein fractions in gluten. For example, $2 \mathrm{~S}$ is a water soluble 
a)

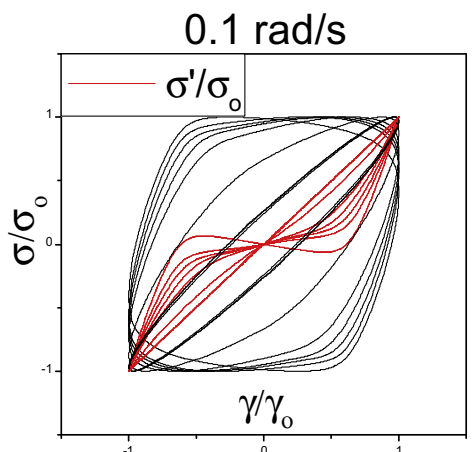

b)

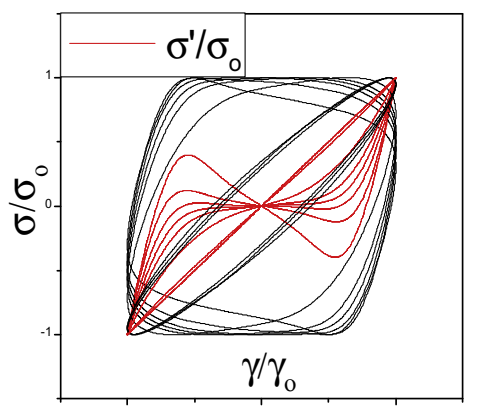

c)

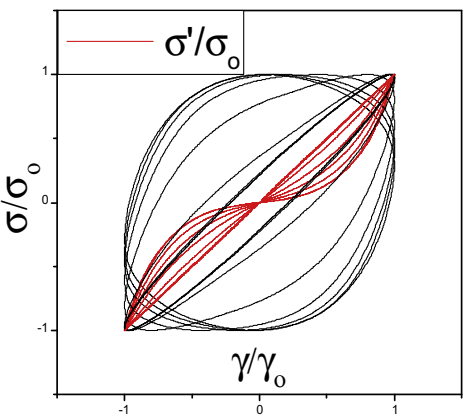

d)

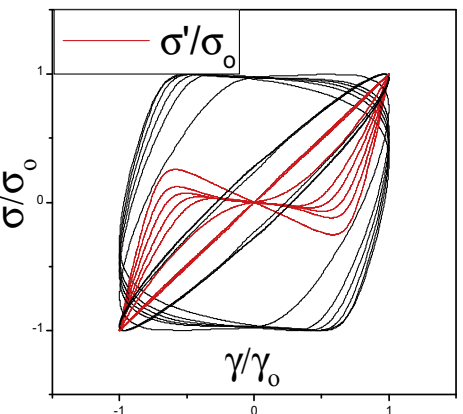

e)

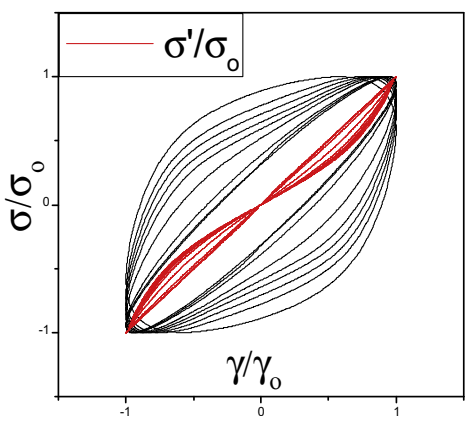

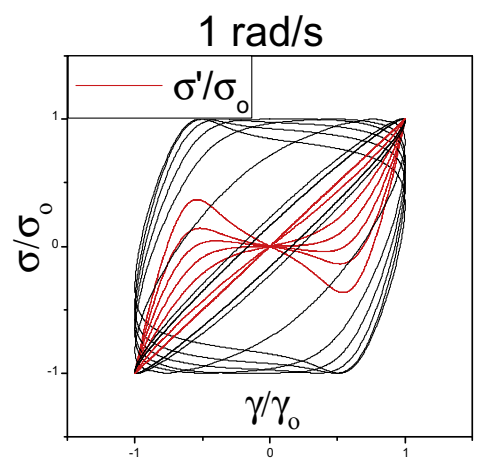
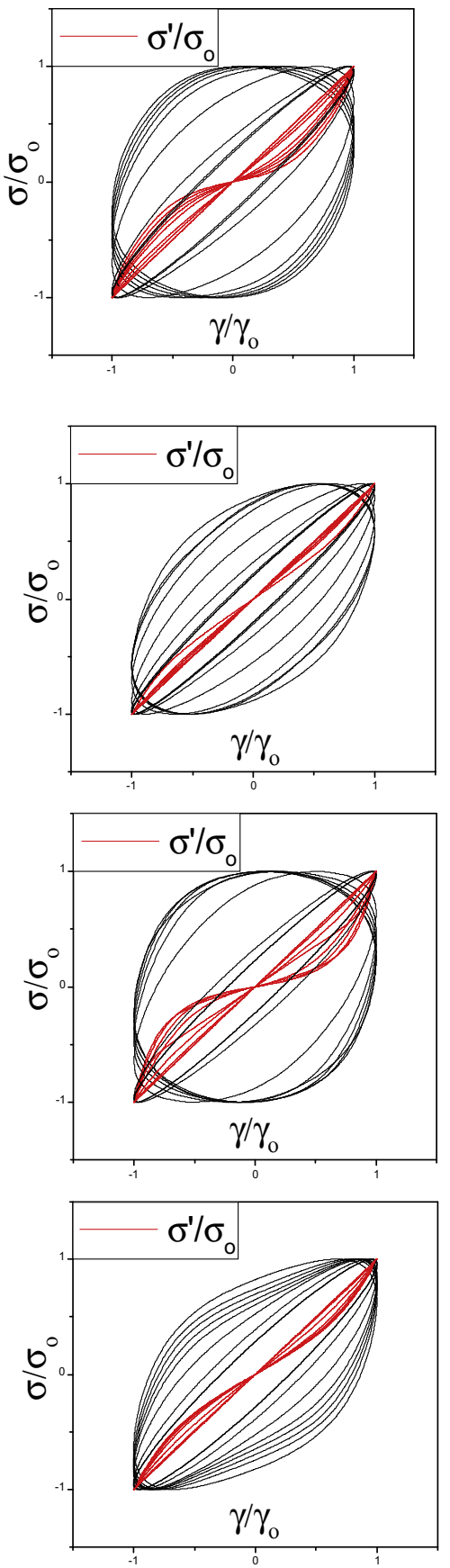
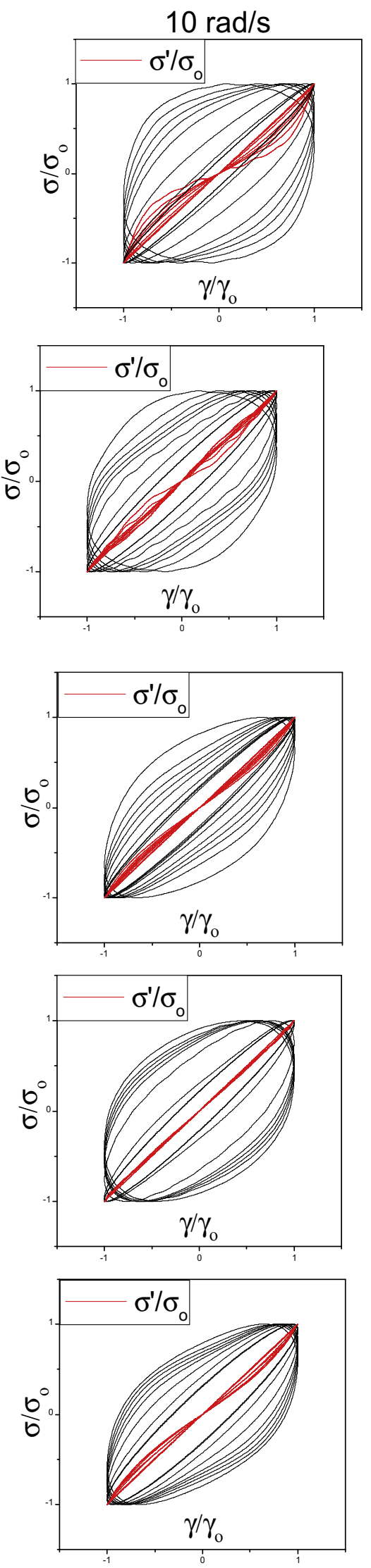

Fig. 2. Lissajous-Bowditch curves for the elastic component of the gluten-free flour dough samples: a) rice flour dough, b) buckwheat flour dough, c) soy flour dough, d) quinoa flour dough, e) soft wheat flour dough. 

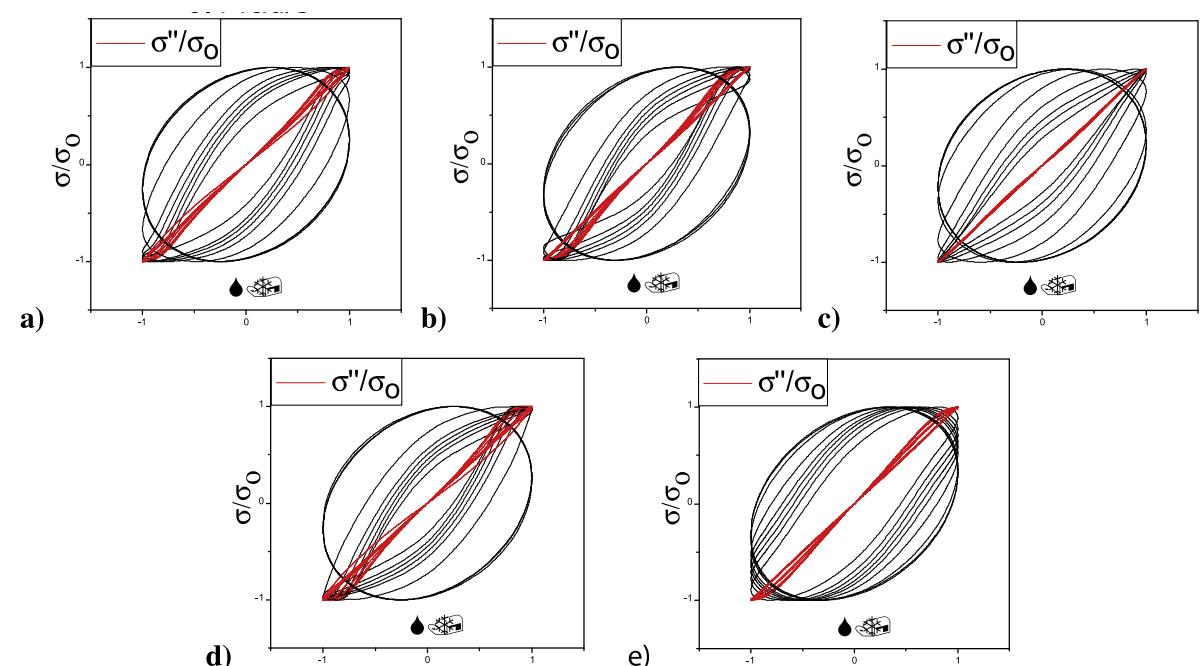

e)

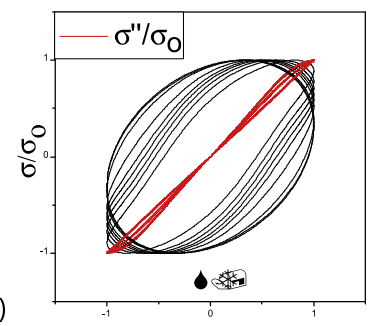

Fig. 3. Lissajous-Bowditch curves for the viscous component of the gluten-free flour dough samples: a) rice flour dough, b) buckwheat flour dough, c) soy flour dough, d) quinoa flour dough, e) soft wheat flour dough at $0.1 \mathrm{rad} / \mathrm{s}$.

globular protein similar to the albumins and globulins in wheat flour, 7S consists of a protein which is cross-linked intramolecularly by disulfide bonds similar to gliadin and finally $11 \mathrm{~S}$ contains 20 times more disulfide bonds with both inter and intramolecular disulfide bonds (Morales and Kokini, 1997) generating its networking ability similar to wheat protein. On the other hand, the protein contents of rice (6-8\%), buckwheat $(\sim 12 \%)$ and quinoa ( $15 \%$ ) flour samples have a much lower protein content compared to soy flour ( 46\%) (Osborne and Mendel, 1917; Ranhotra et al., 1993; Torbica et al., 2010). Because of this contrast, buckwheat and quinoa doughs showed more viscously dominated behavior compared to soy flour but when quinoa flour dough is compared to buckwheat flour dough we observe that quinoa shows more elastic behavior compared to buckwheat at the highest frequency applied. But the elastic component of quinoa dough was affected by the decrease in the frequency more than buckwheat dough. So, the difference in the non-linear behavior of the elastic components of these samples almost disappeared at lower frequencies.

The elastic components $\left(\sigma^{\prime}\right)$, seen as red lines in the loops (Fig. 2), of gluten free dough samples showed strong non-linearities especially observed for buckwheat, quinoa and rice flour dough at $0.1 \mathrm{rad} / \mathrm{s}$ and at high strains. Rice flour dough has similar nonlinearities at $1 \mathrm{rad} / \mathrm{s}$, however, the other dough samples showed more elastic behavior. The non-linearities seen at low frequencies are related to viscous characteristics (Ewoldt et al., 2008). When frequency is low enough, a viscoelastic material would be dominated by its viscous characteristics. These nonlinearities result in secondary loops (self-intersection) in the viscous perspective which will be discussed in the next section. The elastic stress of quinoa dough is almost a straight line even at high strains at $10 \mathrm{rad} /$ $s$ due to the linear viscoelastic behavior which extends to much higher strains at this frequency and one would expect to observe elastically dominated behavior at the higher frequencies such as10 rad/s.

\subsubsection{Viscous component}

For the viscous component the unusual feature of Lissajous curves is the prominent secondary loops. The outer loops represent the linear region (in contrast to the elastic analysis where the inner loops represent the linear analysis) and the inner loops represent the non-linear region. The viscous components of the gluten-free dough samples show the same behavior at the lowest strain value
(0.01\%) at all applied frequencies. The effect of increasing strain became more pronounced as the frequency decreased. In order to focus on the most significant differences we are only showing the viscous Lissajous curves at a frequency of 0.1 radians second where all observed phenomena are magnified. At this frequency once the samples reached the critical $1.6 \%$ strain value, a sharp change was observed leading to the characteristic secondary loops. The viscous component of the soft wheat flour dough represented the most stable behavior when compared to the viscous components of gluten-free dough samples. The behavior of all the flours were considerably different at the frequency of $0.1 \mathrm{rad} / \mathrm{s}$. In the nonlinear region, the order of increasing viscous behavior was as follows; rice dough $>$ buckwheat dough $>$ quinoa dough $>$ soy dough > soft wheat flour dough.

The emergence of secondary loops is due to repeated values at constant strain rate while strain values are different (Ewoldt and McKinley, 2010). This means stress response is independent of instantaneous strain and elastic stress at this particular point must be zero. This behavior is related to a strong nonlinearity in elasticity and there needs to be a partial reversible structural change depending on the time scale of deformation which reoccurs periodically. The origin of this distinctive behavior has been reported to be aging and/or thixotropy (Duvarci et al., 2016).

\subsection{Evaluation of elastic and viscous LAOS parameters of gluten- free dough samples}

The ratio of the third order elastic Chebyshev coefficient to the first order elastic Chebyshev coefficient $\left(e_{3} / e_{1}\right)$ offers insights about intracycle strain stiffening or softening behavior in the non-linear region. It refers to the solid-like character of the material and conceptually it is equivalent to $G^{\prime}$ in small amplitude properties. In fact at the limit of linear behavior $e_{3} / e_{1}=0$ and $e_{1}=G^{\prime}$. Consequently $e_{3} / e_{1}$ is the deviation from linear behavior caused by the need to simulate the distorted stress wave with multiple oscillatory functions using Fourier transforms leading to the third harmonic. All gluten-free dough samples showed higher $e_{3} / e_{1}$ values at the lowest frequency $(0.1 \mathrm{rad} / \mathrm{s})$, except for rice dough. For rice dough $e_{3} / e_{1}$ values increased when the frequency decreased to $1 \mathrm{rad} / \mathrm{s}$ from $10 \mathrm{rad} / \mathrm{s}$, then again decreased when frequency kept decreasing to $0.1 \mathrm{rad} / \mathrm{s}$. For the rest of the dough samples $e_{3} / \mathrm{e}_{1}$ values increased gradually as the frequency decreased. Rice, 
buckwheat, and quinoa dough samples showed higher strain stiffening when compared to soy dough and wheat flour dough and this may be due to starch playing the dominant role in the deformation and stiffening behavior and the poor deformation quality of the protein in these gluten free flour doughs. It must also be noted that there are other components whose significance is much more difficult to judge. For example fat and fiber play a role in affecting rheology and would most certainly play some role in intracycle rheology. Rice contains a small amount of lipids (0.3\%) and also a small amount of fiber $(0.1-0.2 \%)$; buckwheat contains $2-3 \%$ lipids, $3 \%$ fiber; defatted soy flour contains $1 \%$ lipids, 2-6\% fiber; quinoa contains $4-5 \%$ lipids, $8-9 \%$ fiber; and finally wheat flour has 2-2.5\% lipids and 0.5\% fiber (Ranhotra et al., 1993; Mashayekh et al., 2008; Torbica et al., 2010; Pareyt et al., 2011).

The change in strain stiffening with respect to frequency in the soft wheat sample was significantly lower compared to the glutenfree samples. As the frequency decreased, the increase in strain stiffening behavior became more prominent in gluten-free dough samples. Only soy dough showed comparatively similar strain stiffening behavior to the soft wheat flour dough. Fig. 4a shows that rice and soy doughs showed a continuous strain stiffening behavior. The degree of strain stiffening was relatively higher for rice dough which we attribute to the high content of starch. Quinoa dough also showed strain stiffening where the intensity of the stiffening was the lowest among all the flours $\left(\mathrm{e}_{3} / \mathrm{e}_{1} \sim 0\right)$. Buckwheat dough showed $S$ shaped strain stiffening/softening behavior first starting with sharp strain stiffening $\left(e_{3} / e_{1}>0\right)$ followed by softening $\left(e_{3} / e_{1}<0\right)$ and then followed by strain stiffening again. None of the gluten-free doughs showed gradually decreasing strain stiffening behavior observed for the soft wheat flour dough, which may be related to the high loaf volume since the wheat flour dough may deform (extend) more rapidly during expansion. When the Lissajous curves show an elliptical shape, the slope of the tangent at zero strain gives the minimum amplitude modulus $G_{M}^{\prime}$ and the slope of the secant at the max. strain gives the large amplitude modulus $G_{L}^{\prime}$ (Ewoldt et al., 2008). These parameters define the point elastic moduli in LAOS. $G_{L}^{\prime}$ was the highest for rice dough at low strains but at higher strains it was lower than the rest of the doughs (Fig. 4b). A complete comparison of $G_{L}^{\prime}$ and $G_{M}^{\prime}$ values as a function of strain are shown in Fig. 4 b. The high level of starch in rice flour $(\sim 80 \%)$ compared to all the other flours and the interparticle friction at low level of protein results in dilatant behavior and we believe is the origin of this contrarian behavior. For protein rich soy dough which was also the most elastic materials we observe the greatest stability in the values of $G_{L}^{\prime}$ and $G_{M}^{\prime}$.

The stiffening ratio $S$ is given by: $S \equiv\left(G_{L}^{\prime}-G_{M}^{\prime}\right) / G_{L}^{\prime}$ (Ewoldt et al, 2008). Intracycle strain stiffening occurs if $S>0$ and $S<0$ corresponds to intracycle strain softening. $S$ values increased with decreases in frequency. The lowest $S$ values were observed for wheat flour dough and soy dough samples. $S$ values were $<1$ for soy dough and wheat flour dough. The rest of the gluten-free dough samples showed values $>1$ at the lowest frequency. All gluten-free dough samples showed strain stiffening behavior in the non-linear region at all applied frequencies (data not shown).

\subsection{Viscous properties of gluten-free dough samples}

The ratio of the third order viscous Chebyshev coefficient to the first order viscous Chebyshev coefficient $\left(v_{3} / v_{1}\right)$ is one of the LAOS parameters used to explain the non-linear behavior for the viscous component. This is conceptually similar to $G^{\prime \prime}$ in small amplitude oscillatory properties. The major difference is that $\mathrm{G}^{\prime \prime}$ offers no information related to intracycle behavior. The intracycle viscous non-linear property shows shear thickening when $v_{3} / v_{1}>0$ and as shear thinning when $v_{3} / v_{1}<0$ (Ewoldt et al., 2008). All the gluten- free dough samples and the control showed shear thinning behavior at the highest strains and at all the frequencies applied (Fig. 4a). The thickening ratio $\mathrm{T}$ is given by $T \equiv\left(\eta_{L}-\eta_{M}\right) / \eta_{L} . T>0$ corresponds to intracycle shear thickening, whereas $T<0$ shows shear thinning (Ewoldt et al., 2008). T values also suggested that the gluten-free dough samples showed shear thinning behavior in the non-linear region due to the negative values of $\mathrm{T}$.

The large rate dynamic viscosity, $\eta_{L}^{\prime}$, represents the instantaneous viscosity at the amplitude value of shear rate during the oscillatory deformation. The minimum rate dynamic viscosity $\eta_{M}^{\prime}$ is the intracycle viscosity at the lowest dynamic shear rate. Rice and buckwheat dough samples showed the lowest $\eta_{M}^{\prime}$ values, whereas the highest values were observed for soy and then soft wheat flour dough at $10 \mathrm{rad} / \mathrm{s}$. The largest values for $\eta_{L}^{\prime}$ were observed for soy dough. As the frequency decreased, $\eta_{L}^{\prime}$ values increased for all the dough samples. Rice dough was strongly frequency dependent and showed the highest value at the lowest frequency applied $(0.1 \mathrm{rad} /$ s). Buckwheat and quinoa dough samples showed similar values while soy dough showed similar values compared to soft dough.

\subsection{Correlation of LAOS data with baking volume}

The loaf volumes and crumb properties of the gluten-free bread samples and soft wheat bread can be seen in Fig. 5. Correlations between selected LAOS parameters and the loaf volume are evaluated. We focus particularly on $G_{L}^{\prime}$ and $G_{M}^{\prime}$ because they relate to the non linear elasticity in the sample representing the stretching properties in the doughs that may have the most influence on loaf volume development based on prior understanding (Huang and Kokini, 1993).

In fact the best correlation among the elastic LAOS parameters and the bread volume was obtained with $G_{L}^{\prime}$ values $\left(\mathrm{R}^{2}=0.58\right)$ and $G_{M}^{\prime}\left(\mathrm{R}^{2}=0.74\right)$ at a frequency of $10 \mathrm{rad} / \mathrm{s}$ (Fig. 6). Correlations at other strains and frequencies were much worse. There appears to be an imperfect and mild correlation between increasing $G_{L}^{\prime}$ and $G_{M}^{\prime}$ values with increasing loaf volume. We attribute the mildly high correlation at this frequency and strain to the fact hat as the bubbles grow during baking the biaxial extension rate is considerable although we don't have quantitative estimates for this value. However, too high $G_{L}^{\prime}$ values and the strain stiffening, which is observed as in the case of rice dough, caused a lower loaf volume relative to soft wheat flour, soy, quinoa, buckwheat flour doughs. Consequently, $G_{L}$ is also not a perfect marker but it enables to help us think through the possible mechanisms involved in loaf volume development. Since $G_{L}^{\prime}$ and $G_{M}^{\prime}$ are imperfect markers strain stiffening/softening properties were used to augment and complement the information they provide. The non-linear rheological property which is useful here is $e_{3} / e_{1}$, a measure of strain stiffening/softening. We observe that the largest strain stiffening occurs (largest positive values) with rice flour during intracycle deformation. This observation may explain why high strain stiffening behavior results in lower bread volume for rice bread. Strain stiffening followed by strain softening behavior on the other hand results in increased bread volume since after a critical strain value the cells can continue to grow as observed with soft wheat flour. In the rest of the gluten free flours strain stiffening is modest as in the case of quinoa and buckwheat flours. For soy flour it appears that strain stiffening increases but not to the degree of precluding cell growth. At this point in our understanding we believe we have developed a uniquely accurate qualitative view but we are unable to offer a quantitative model which correlates well with loaf volume. This will be the subject of future research. During fermentation, the $\mathrm{CO}_{2}$ produced by the yeast in the aqueous phase saturates the water as fermentation proceeds. Once the water is saturated, $\mathrm{CO}_{2}$ starts expanding the dough bubbles because of the increased pressure. 


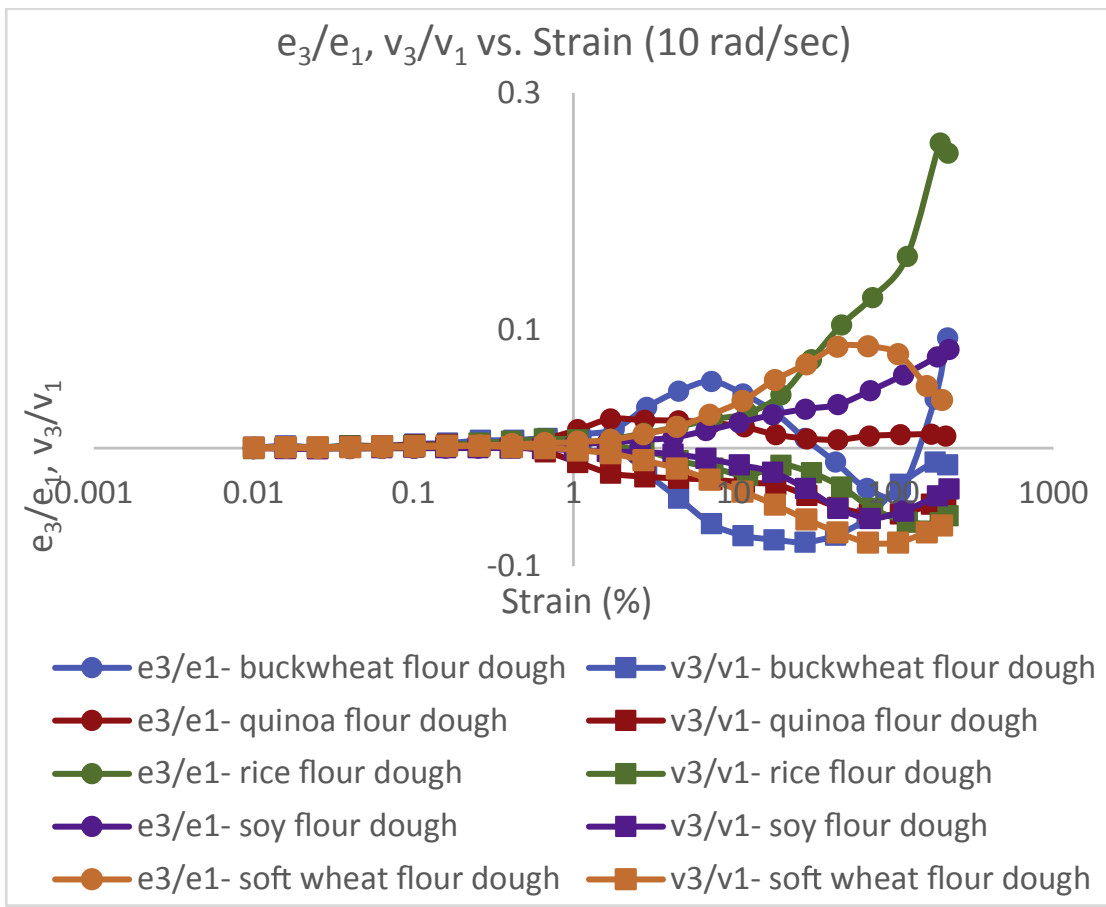

a)

b)

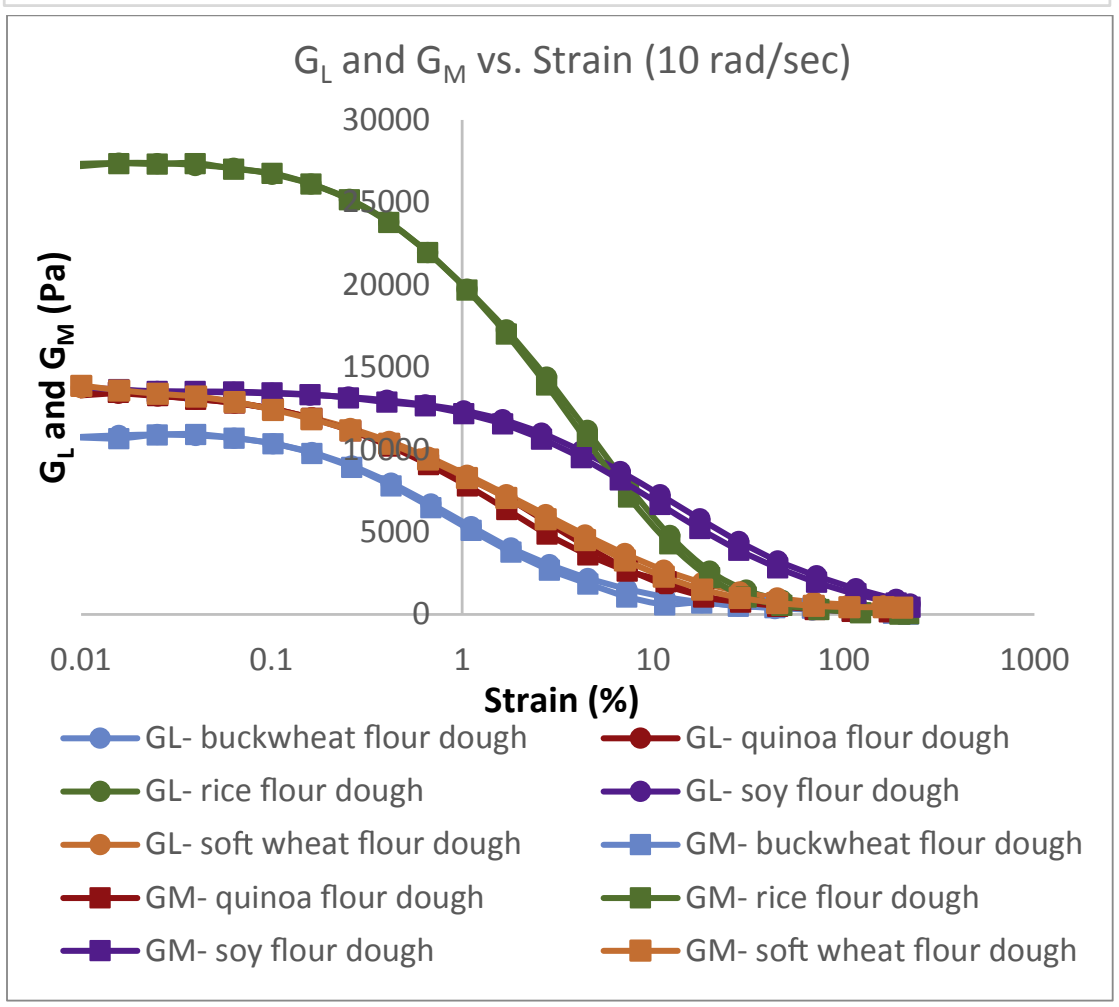

Fig. 4. Elastic LAOS parameters at $10 \mathrm{rad} / \mathrm{s}$ frequency for gluten-free dough samples in comparison with soft wheat flour dough: a) $e_{3} / e_{1}$ values, b) $G_{L}$ values.

Dough's viscoelastic properties resist the bubble expansion (Huang and Kokini, 1993) and helps stabilizing the bubble size. As a result, the volume of the dough mass is increased, as the dough is leavened (Delcour and Hoseney, 2010). If the dough structure is too stiff, the pressure of the $\mathrm{CO}_{2}$ is not enough to extend and expand the network resulting in a decrease in volume. So, the dough structure should be neither so weak, nor so stiff for a desired gas cell size, distribution, and loaf volume. The optimum behavior is described by a balance between strength and extensibility in dough. Increasing strain stiffening behavior above the optimum results in high entanglement network density that may cause a decrease in loaf volume (Sroan et al., 2009). It must be kept in mind that rheology is only one of the factors involved in bubble growth and bubble setting (Kokini et al., 1992; Huang and Kokini, 1993) and in fact biaxial extensional viscosity is the most significant rheological property which is linked with shear rheology using Trouton's ratio. 


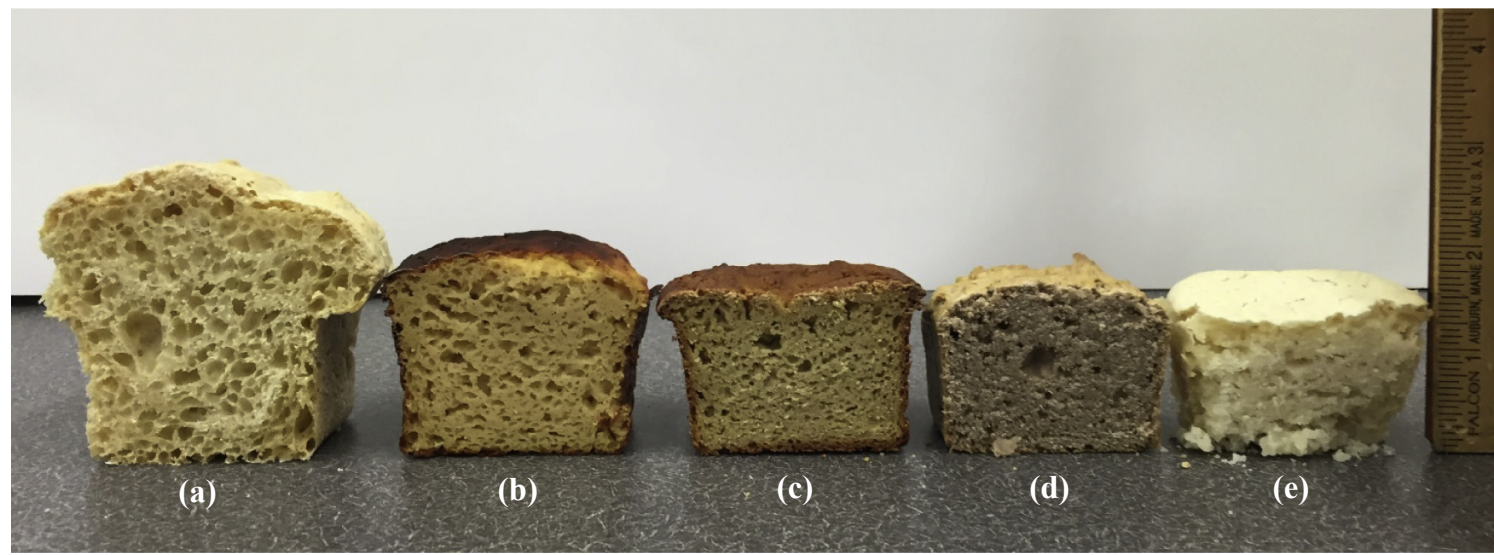

Fig. 5. a) wheat bread, b) soy flour bread, c) quinoa flour bread, d) buckwheat flour bread, e) rice flour bread.
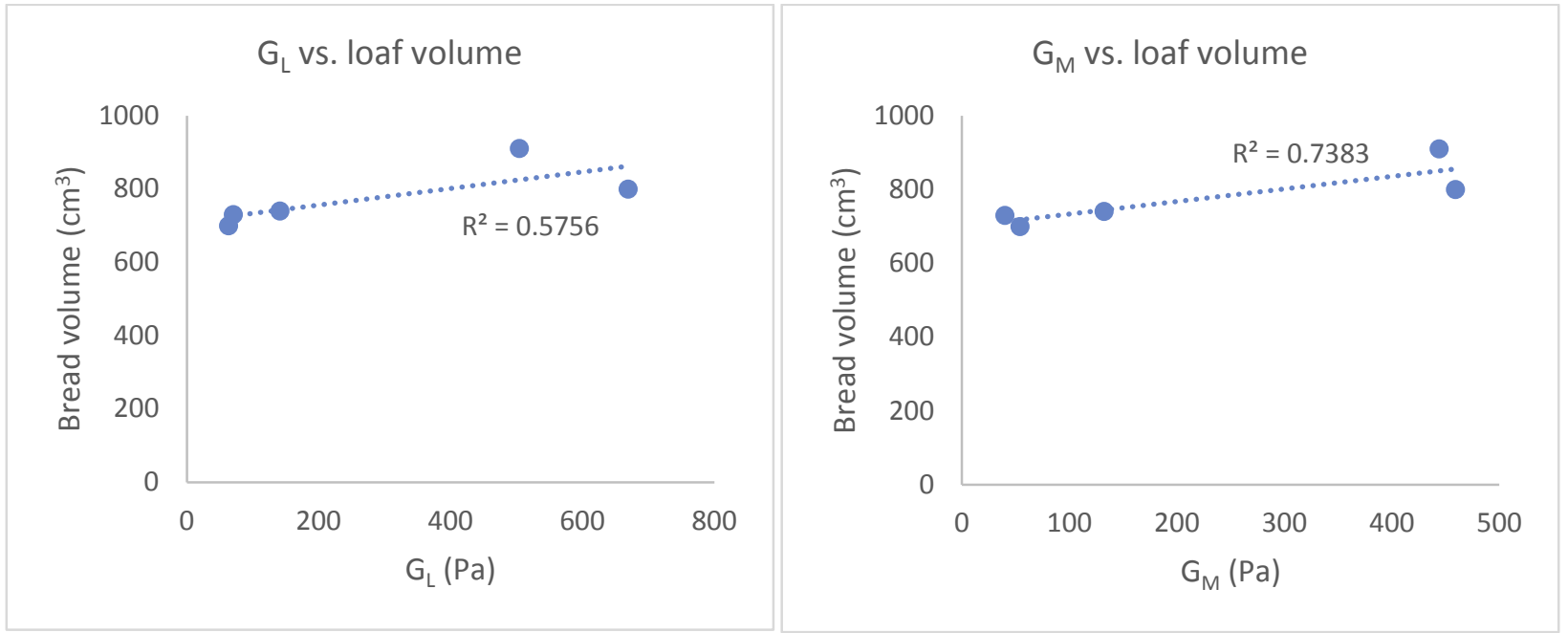

Fig. 6. Trendlines for the correlations between LAOS parameters and the gluten-free bread volume.

The effective Trouton ratio for each dough may be very different. In addition, nucleation properties (Moraru and Kokini, 2003) are another major factor influencing expansion and they may be considerably different for each dough. The totality of these factors explains why each formulation has a different loaf volume.

\section{Conclusion}

Gluten-free flours were evaluated using LAOS in comparison with soft wheat flour dough. The elastic Lissajous curves showed that soy dough gave the most intracycle elasticity, while rice dough gave the least intracycle elasticity among the gluten-free doughs. As the frequency decreased, all dough samples started to show more viscously dominated viscoelastic behavior in the non-linear region. The viscous behavior was more pronounced in rice, buckwheat, and quinoa dough. At the highest frequency applied, soy dough showed quite similar elastic Lissajous curves to those of soft wheat flour dough. Lissajous curves also showed that both elastic and viscous components of all gluten-free dough samples were strongly dependent on strain. The LAOS parameters $G_{\mathrm{L}}^{\prime}, G_{\mathrm{M}}^{\prime}$ showed a significant but soft correlation with bread loaf volume. LAOS test is a useful new technique to determine the non-linear intracycle properties of gluten free dough materials which have never been studied before. The new intracycle information in the non-linear region may be more relevant to processing where the applied strains are very large. Thus, the new parameters obtained may be useful to improve the gluten-free formulations and gluten-free baked products with better quality may be developed.

\section{References}

AACC, 2000. Farinograph Method for Flour. AACC Method 54-21. Approved Methods of the AACC, vol. II. American Association of Cereal Chemists, Inc., USA Amemiya, J.L. Menjivar, J.A., 1992. Comparison of small and large deformation measurements to characterize the rheology of wheat flour doughs. J. Food Eng. 16 (1-2), 91-108.

Bagley, E.B., Dintzis, F.R., Chakrabarti, S., 1998. Experimental and conceptual problems in the rheological characterization of wheat flour doughs. Rheol. Acta 37, 556-565.

Crockett, R., le, P., Vodovotz, Y., 2011. Effects of soy protein isolate and egg white solids on the physicochemical properties of gluten-free bread. Food Chem. 129, 84-91.

Delcour, J.A., Hoseney, R.C., 2010. Principles of Cereal Science and Technology. AACC International, Inc., St. Paul, MN.

Demirkesen, I., Mert, B., Sumnu, G., Sahin, S., 2010. Utilization of chestnut flour in gluten-free bread formulations. J. Food Eng. 101, 329-336.

Dus, S.J., Kokini, J.L., 1990. Prediction of the nonlinear viscoelastic properties of a hard wheat flour dough using the Bird-Carreau constitutive model. J. Rheol. 34 (7), 1069-1084

Duvarci, O.C., Yazar, G., Kokini, J.L., 2016. The comparison of LAOS behavior of structured food materials (suspensions, emulsions and elastic networks). Trends Food Sci. Technol. xxx, 1-10 (in press).

Ewoldt, R.H., Hosoi, A.E., McKinley, G.H., 2007. Rheological fingerprinting of complex fluids using large amplitude oscillatory shear (LAOS) flow. Annu. Trans. 
Nord. Rheol. Soc. 15, 3-8.

Ewoldt, R.H., Hosoi, A.E., McKinley, G.H., 2008. New measures for characterizing nonlinear viscoelasticity in large amplitude oscillatory shear. J. Rheol. 52 (6), 1427-1458.

Ewoldt, R., McKinley, G.H., 2010. On secondary loops in LAOS via self-intersection of Lissajous-Bowditch curves. Rheol. Acta 49, 213-219.

Faubion, J.M., Hoseney, R.C., 1990. The viscoelastic properties of wheat flour doughs. In: Faridi, H., Faubion, J.M. (Eds.), Dough Rheology and Baked Product Texture. Van Nostrand Reinhold, New York, pp. 29-67.

Gallagher, E., Kunkel, A., Gormley, T.R., Arendt, E.K., 2003. The effect of dairy and rice powder addition on loaf and crumb characteristics, and on shelf life (intermediate and long-term) of gluten-free breads stored in a modified atmosphere. Eur. Food Res. Technol. 218, 44-48.

Gularte, M.A., de la Hera, E., Gómez, M., Rosell, C.M., 2012. Effect of different fibers on batter and gluten-free layer cake properties. Food Sci. Technol. LEB 48, 209-214.

Huang, H., Kokini, J.L., 1993. Measurement of biaxial extensional viscosity of wheat flour doughs. J. Rheol. 37 (5), 879-891.

Janssen, A.M., van Vliet, T., Vereijken, J.M., 1996. Fundamental and empirical rheological behavior of wheat flour doughs and comparison with bread making performance. J. Cereal Sci. 23, 43-54.

Kokini, J.L., Chang, C.N., Lai, L.S., 1992. The role of rheological properties on extrudate expansion. In: Kokini, J.L., Ho, C.T., Karwe, M. (Eds.), Food Extrusion Science and Technology. Marcel Dekker NY, pp. 631-652.

Lazaridou, A., Duta, D., Papageorgiou, M., Belc, N., Biliaderis, C.G., 2007. Effects of hydrocolloids on dough rheology and bread quality parameters in gluten-free formulations. J. Food Eng. 79, 1033-1047.

Mashayekh, M., Mahmoodi, M.R., Entezari, M.H., 2008. Effect of fortification of defatted soy flour on sensory and rheological properties of wheat bread. Int. J.
Food Sci. Tech. 43 (9), 1693-1698.

Morales, A., Kokini, J.L., 1997. Glass transition of soy globulins using differential scanning calorimetry and mechanical spectrometry. Biotechnol. Prog. 13, 624-629.

Moraru, C.I., Kokini, J.L., 2003. Nucleation and expansion during extrusion and microwave heating of cereal foods. Compr. Rev. Food Sci. Food Saf. 2 (4) $142-165$.

Nunes, M.H.B., Moore, M.M., Ryan, L.A.M., Arendt, E.K., 2009. Impact of emulsifiers on the quality and rheological properties of gluten-free breads and batters. Eur. Food Res. Technol. 228, 633-642.

Osborne, T.B., Mendel, L.B., 1917. The use of soybean as food. J. Biol. Chem. 32 369-387.

Pareyt, B., Finnie, S.M., Putseys, J.A., Delcour, J.A., 2011. Lipids in bread making: sources, interactions, and impact on bread quality. J. Cereal Sci. 54, 266-279.

Phan-Thien, N., Safari-Ardi, M., Morales-Patino, A., 1997. Oscillatory and simple shear flows of a flour-water dough: a constitutive mode. Rheol. Acta 36, 38-48.

Ranhotra, G.S., Gelroth, J.A., Glaser, B.K., Lorenz, K.J., Johnson, D.L., 1993. Composition and protein and nutritional quality of quinoa. Cereal Chem. 70 (3) 303-305.

Sroan, B.S., Bean, S.R., MacRitchie, F., 2009. Mechanism of gas cell stabilization in bread making. I. The primary gluten-starch matrix. J. Cereal Sci. 49, 32-40.

Torbica, A., Hadnadev, M., Dabcevic, D., 2010. Rheological, technological, and sensory properties of gluten-free bread formulations based on rice and buckwheat flour. Food Hydrocoll. 24, 626-632.

Witczak, M., Korus, J., Ziobro, R., Juszczak, L., 2010. The effects of maltodextrins on gluten-free dough and quality of bread. J. Food Eng. 96, 258-265.

Yazar, G., Duvarci, O., Tavman, S., Kokini, J.L., 2016. Non-linear rheological properties of soft wheat flour dough at different stages of Farinograph mixing. Appl. Rheol. $26,1-11$. 